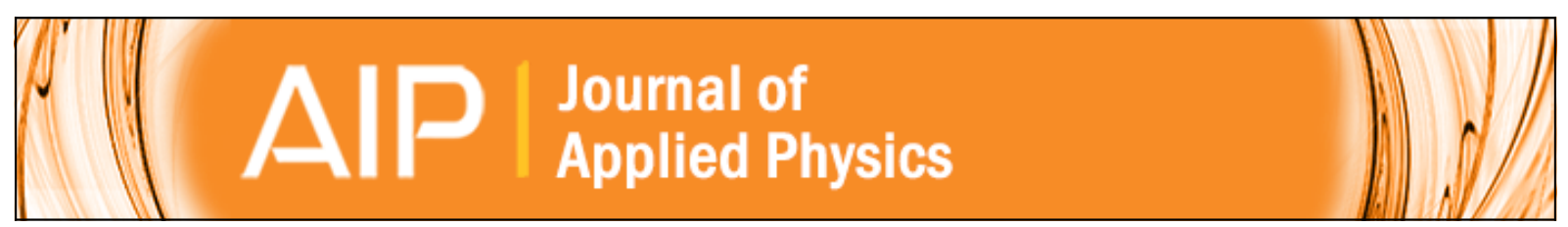

\title{
A new adsorption substrate: Single crystal exfoliated graphite
}

Roy Clarke, P. M. Horn, S. E. Nagler, and T. F. Rosenbaum

Citation: Journal of Applied Physics 55, 1231 (1984); doi: 10.1063/1.333171

View online: $\mathrm{http}: / / \mathrm{dx}$.doi.org/10.1063/1.333171

View Table of Contents: http://scitation.aip.org/content/aip/journal/jap/55/4?ver=pdfcov

Published by the AIP Publishing

\section{Articles you may be interested in}

Superhydrophilic graphite surfaces and water-dispersible graphite colloids by electrochemical exfoliation

J. Chem. Phys. 139, 064703 (2013); 10.1063/1.4817680

Single heterojunction solar cells on exfoliated flexible $25 \mathrm{~m}$ thick mono-crystalline silicon substrates

Appl. Phys. Lett. 102, 163904 (2013); 10.1063/1.4803174

Thermal exfoliation and crystallographic transformation of single-crystal metal oxides induced by He-ion implantation

J. Appl. Phys. 94, 3045 (2003); 10.1063/1.1600525

Gas Adsorption by Refractory Metal Single Crystals

J. Vac. Sci. Technol. 9, 1241 (1972); 10.1116/1.1317019

Adsorption of Gases on Quartz Single Crystals

J. Chem. Phys. 36, 2688 (1962); 10.1063/1.1732353

\section{A|P $\left.\right|_{\text {Applied Physics }} ^{\text {Journal of }}$}

Journal of Applied Physics is pleased to

announce André Anders as its new Editor-in-Chief 


\title{
A new adsorption substrate: Single crystal exfoliated graphite
}

\author{
Roy Clarke \\ Department of Physics, The University of Michigan, Ann Arbor, Michigan 48109 \\ P. M. Horn, S. E. Nagler, and T. F. Rosenbauma) \\ IBM Thomas J. Watson Research Center, Yorktown Heights, New York 10598
}

(Received 5 August 1983; accepted for publication 2 November 1983)

\begin{abstract}
We describe the controlled exfoliation of single crystal graphite flakes intercalated to stage eleven with $\mathrm{SbCl}_{5}$. The resulting exposed surface remains well oriented in the basal plane. Our best sample to date has an internal surface area of $6 \mathrm{Torr} c \mathrm{c}$ and an in-plane mosaic of $2.3^{\circ} \mathrm{HWHM}$ (half-width at half-maximum). High resolution x-ray scattering from adsorbed krypton reveals an average step-free surface size of at least $2000 \AA$. Experiments which probe orientational information in the layer planes should now be possible.
\end{abstract}

PACS numbers: 81.20. $-\mathrm{n}, 68.20 .+\mathrm{t}$, 06.60.Ei, 68.55. $+\mathrm{b}$

Synthetic graphite (e.g., Union Carbide HOPG) ${ }^{1}$ has provided the basis for studies on a broad range of physical systems. ${ }^{2}$ For example, it is commonly used as a matrix for intercalation and as a substrate material for catalysis and adsorption experiments. Although samples of synthetic graphite can be prepared with much larger volumes than natural graphite flakes, they are oriented only along the $c$ axis, precluding detailed structural analysis of adsorbed or intercalated layers.

In this communication, we describe a procedure to exfoliate single crystals of graphite, resulting in a well-oriented substrate with high specific surface area. This substrate makes possible new classes of experiments in fields as diverse as superconductivity, two-dimensional melting, ${ }^{3}$ magnetism, and commensurate-incommensurate transitions.

The exfoliation procedure is as follows. Single crystals of natural graphite (typically $6 \times 6 \times 0.2 \mathrm{~mm}^{3}$ ) were carefully intercalated to Stage $11^{5}$ with $\mathrm{SbCl}_{5}$. The two-bulb vapor intercalation technique ${ }^{6}$ was used, with the graphite held at $200{ }^{\circ} \mathrm{C}$ and the liquid $\mathrm{SbCl}_{5}$ at $40^{\circ} \mathrm{C}$. This particular intercalant is chosen for several reasons. Firstly, its graphite intercalation compound (GIC) is unusually stable $e^{6}$ in ambient atmosphere and is therefore easy to handle. Secondly, the intercalant gallery spacing, at $\sim 9.4 \AA,{ }^{7}$ is among the largest encountered in well-staged GIC's. This is undoubtedly related to the ease with which $\mathrm{SbCl}_{5}$-graphite can be exfoliated. ${ }^{8}$

Intercalation to precisely stage 11 is not crucial for this purpose except that high stage gives an ideal balance between the requirement for a large internal surface area (exposed on deintercalation) and the desire to introduce as few defects ${ }^{9}$ as possible into the graphite layers. For the latter reason we avoided low intercalation stages.

After intercalation, the sample was mounted in a vertical furnace containing an evacuable $\left(\sim 10^{-5}\right.$ Torr) piston and cylinder device, and was slowly heated to $1100^{\circ} \mathrm{C}$. A magnet was attached to the top of the quartz piston, and by means of a solenoid we could vary the compressional force on the surface of the graphite sample from $0-1 \mathrm{~N}$. With the upper limit of compressional force, we could control the exfoliation of samples of initial thickness up to $0.3 \mathrm{~mm}$. The

\footnotetext{
a) Present address: The James Franck Institute, University of Chicago, 5640 Ellis Avenue, Chicago, IL 60637.
}

exfoliation was marked by a sharp ${ }^{10}$ onset at approximately $300^{\circ} \mathrm{C}$ and continued until the sample was $30-50$ times its original thickness, as monitored in situ by a traveling microscope. Until the intercalant starts to escape from the graphite (at $\sim 800^{\circ} \mathrm{C}$ ), the expansion is reversible and both slow heating $\left(1-2^{\circ} \mathrm{C} / \mathrm{min}\right)$ and full compression are required to prevent the sample from exploding or shearing. At temperatures above $800^{\circ} \mathrm{C}$ the quartz piston was lifted from the graphite surface; the sample was baked for $1 \mathrm{~h}$ at $1100^{\circ} \mathrm{C}$ in order to clean it, and, finally, the sample was allowed to cool to ambient in a helium atmosphere.

Electron microprobe experiments on samples baked at $1100^{\circ} \mathrm{C}$ show a small $(1-2$ mole $\%)$ residue of metallic $\mathrm{Sb}$ and this contaminant results in a spurious $\mathrm{x}$-ray diffraction peak at $1.62 \AA^{-1}$. Higher baking temperatures (e.g., $1500^{\circ} \mathrm{C}$ ) would probably eliminate this residue. It is worth pointing out here that other GIC's, such as $\mathrm{HNO}_{3}$-graphite, ${ }^{2}$ have potential advantages in terms of lower process temperatures and less contamination. However, we find that exfoliation proceeds much more rapidly in this case and is harder to control. We have so far been able to exfoliate only very thin $\left(<0.1 \mathrm{~mm}\right.$ ) samples of $\mathrm{HNO}_{3}$-graphite, and these are too flimsy for experimental work.

Figure 1 shows a side view of a $\mathrm{SbCl}_{5}$-exfoliated single crystal $6 \times 6 \times 7 \mathrm{~mm}$ thick. A puckering of the graphite layers can be clearly seen in this photograph. This is caused by the way in which the sample expands during exfoliation: a closer inspection of the puckered surface reveals a network of interconnected "pockets." These probably contain gaseous intercalant in the temperature region between the onset of exfoliation $\left(\sim 300^{\circ} \mathrm{C}\right)$ and deintercalation $\left(\sim 800^{\circ} \mathrm{C}\right)$, suggesting a mechanism for the reversible expansion of the sample. It is interesting to note that the observed expansion behavior and the resulting connectedness of the exfoliated sample are both entirely consistent with the Daumas and Herrold picture ${ }^{11}$ of stage formation in GIC's. In this model, the intercalant is not homogeneously distributed in layers within the matrix but instead forms macroscopic islands between every pair of contiguous carbon layers. This requirement is necessary to explain many features of GIC's, especially the redistribution of intercalant within the matrix. One example where the latter is important is in pressure- 


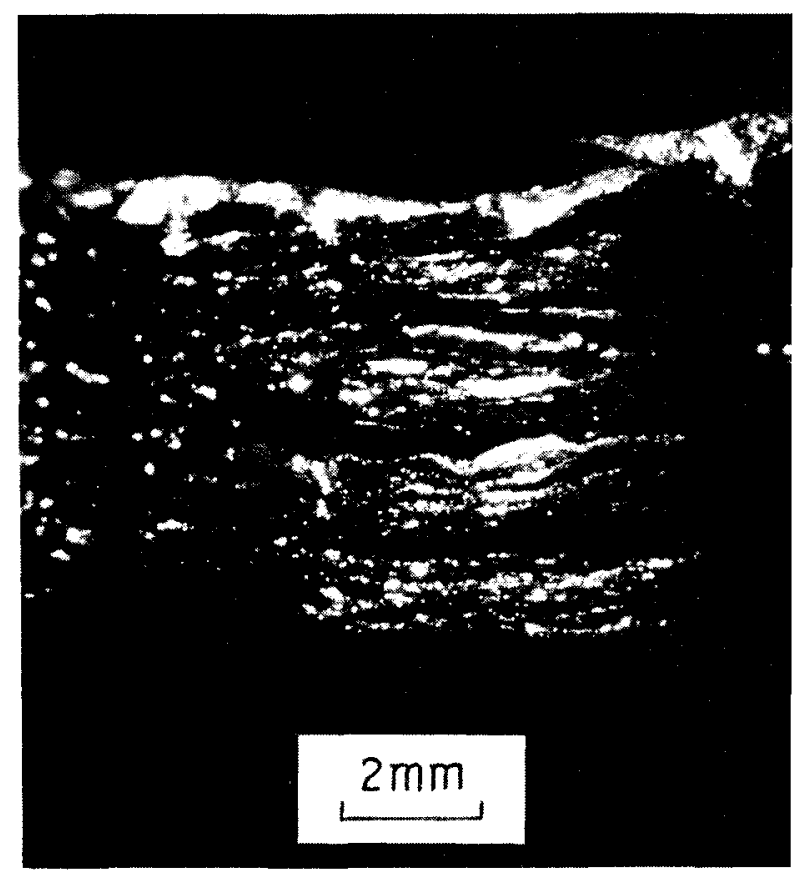

FIG. 1. Optical micrograph of exfoliated graphite crystal; side view.

induced staging transitions. ${ }^{12}$

Our best sample to date has an internal surface area of 6 Torr cc $\left(2 \times 10^{17}\right.$ atoms/adsorbed monolayer) and an inplane mosaic of $2.3^{\circ} \mathrm{HWHM}$. The adsorption isotherm is shown in Fig. 2 and was obtained using the gas handling system and cryostat discussed previously. ${ }^{13}$ Structural characterization was performed with $\mathrm{Cu} K \alpha$ radiation from a $\mathrm{Ri}$ gaku $12 \mathrm{~kW}$ rotating anode source. The $\mathrm{x}$ rays were collimated in-plane with a single-crystal germanium (111) monochromator before the sample, and a Ge (111) crystal analyzer after the sample.

Figure 3 shows in-plane $\omega$ scans through one of the six

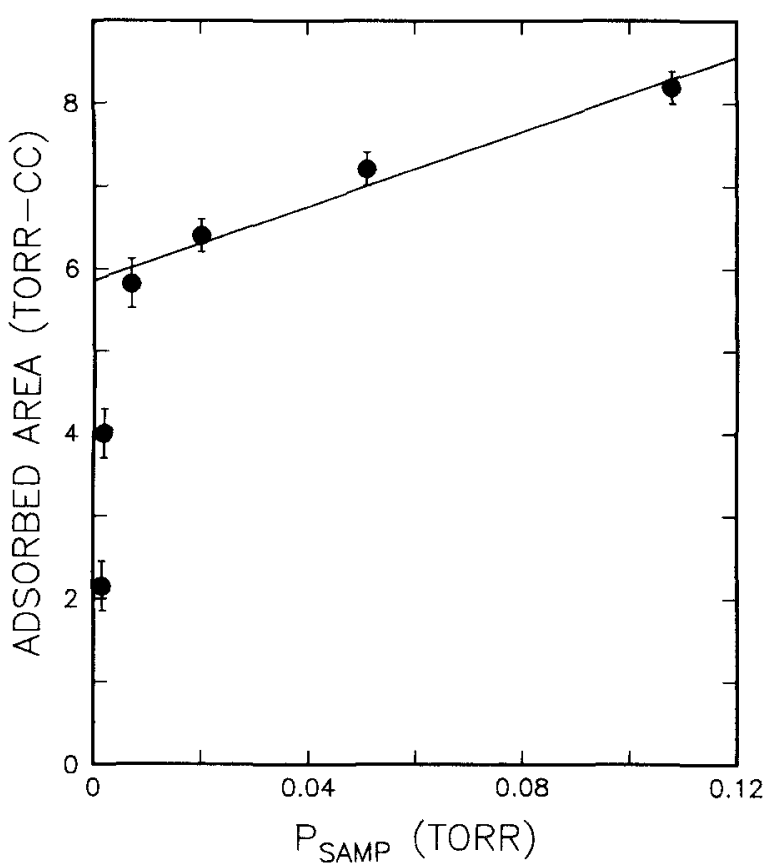

FIG. 2. Krypton adsorption isotherm taken at $T=77 \mathrm{~K}$.

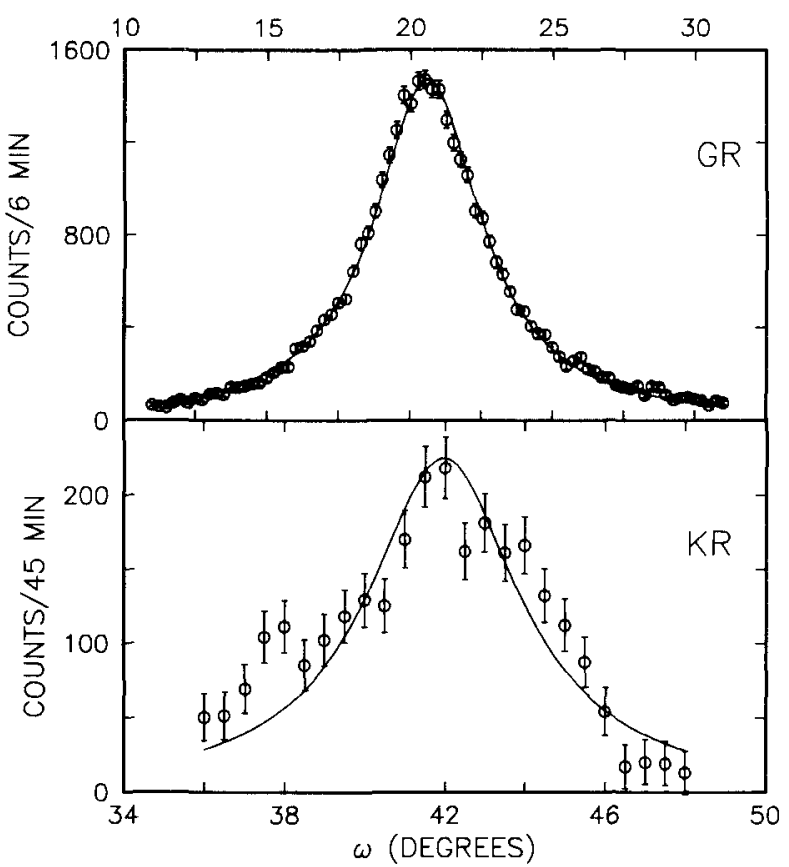

FIG. 3. In-plane $\omega$ scans through (a) graphite (10) peak, (b) primary krypton peak. Solid lines are Lorentzian fits (see text).

graphite $(10)$ spots of the exfoliated crystal and also through the $(\sqrt{3} \times \sqrt{3}) \mathbf{R} 30^{\circ}$ peak of an adsorbed monolayer of krypton. The best fit to the graphite peak is a Lorentzian with $2.3^{\circ}$ HWHM. The scattering from the adsorbed $\mathrm{Kr}$ monolayers is obviously quite weak. The best fit value of the in-plane $\mathrm{Kr}$ mosaic is about $3^{\circ} \mathrm{HWHM}$ although a Lorentzian fit with width constrained to $2.3^{\circ} \mathrm{HWHM}$ (shown in Fig. 3) seems to work adequately. Figure 4 is a radial scan through the same krypton Bragg peak. The solid line corresponds to a lineshape analysis of the data which is essentially identical to

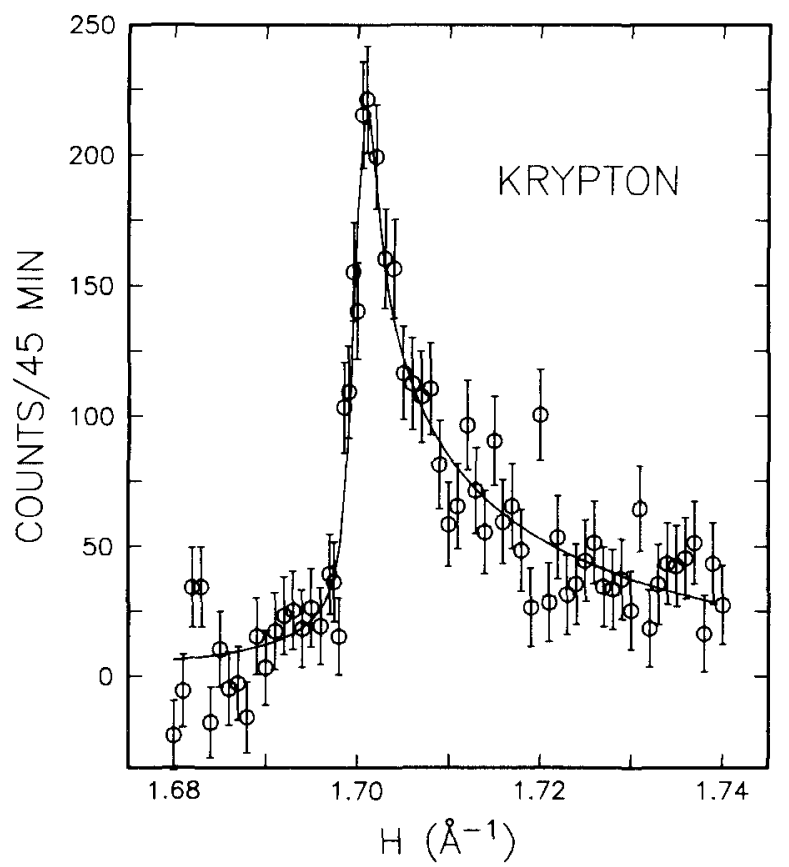

FIG. 4. Bragg peak from krytpon monolayer. The solid line is a Warren fit appropriate to 2 -d scattering. 
that usually carried out for $Z Y X$ graphite substrates ${ }^{14}$ (except that the powder average is replaced by an average over the in-plane mosaic). This fit yields an average step-free surface size of at least $2000 \AA$ and a $c$-axis tilt mosaic of $17^{\circ}$ HWHM. The intensity of this scattering suggests that it originates from $\sim 1000$ separate krypton layers.

In conclusion, $\mathrm{SbCl}_{5}$ seems to be an excellent exfoliant for the preparation of well-oriented natural graphite substrates. The in-plane coherence length (which is comparable to present $Z Y X$ substrates ${ }^{15}$ ), together with the good inplane mosaic, has already made a qualitative improvement in experiments on melting phenomena in adsorbed monolayers. ${ }^{3}$ Single crystal exfoliated graphite should facilitate a broad range of surface science experiments where a microscopically smooth, well-oriented substrate is called for.

We would like to thank J. Anderson, J. Angellelo, W. Haag, and P. Sanda for technical assistance, P. Dimon for help with the data analysis, and $\mathrm{H}$. Homma for preparation of intercalated graphite samples. The work was supported in part by the National Science Foundation Low Temperature Physics Grant DMR-8207449. One of us (S.E.N.) was supported by a Natural Sciences and Engineering Research Council of Canada Fellowship.
'A. W. Moore, Chemistry and Physics of Carbon (Dekker, New York, 1981), Vol. 17, p. 233.

${ }^{2}$ For a general review of the properties of graphite intercalation compounds, see M. S. Dresselhaus and G. Dresselhaus, Adv. Phys. 30, 139 (1981).

${ }^{3}$ T. F. Rosenbaum, S. E. Nagler, P. M. Horn, and Roy Clarke, Phys. Rev. Lett. 50, 1791 (1983).

${ }^{4}$ From a mine near Harrisville, New York.

5The "stage" is defined as the number of carbon layers between intercalant layers.

${ }^{6}$ J. Mélin and A. Hérold, C. R. Acad. Sci. Paris 269, 877 (1969).

'See Ref. 2 for comparison with other GIC's.

${ }^{8} \mathrm{~J}$. Mélin and A. Hérold, Carbon 13, 357 (1975).

${ }^{9}$ M. E. Misenheimer and H. Zabel, Phys. Rev. B 27, 1443 (1983), and references therein.

${ }^{10}$ In-plane $\mathrm{x}$-ray diffraction experiments reveal that the onset of exfoliation is accompanied by an irreversible disordering of the intercalant structure. H. Homma, University of Michigan, Ph.D. thesis, 1984 (unpublished).

"N. Daumas and H. Hérold, C. R. Acad. Sci. Paris 268, 373 (1969).

${ }^{12}$ R. Clarke, N. Wada, and S. A. Solin, Phys. Rev. Lett. 44, 1616 (1980).

${ }^{13}$ P. M. Horn, R. J. Birgeneau, P. A. Heiney, and E. M. Hammonds, Phys Rev. Lett. 41, 961 (1978).

${ }^{14}$ P. A. Heiney, R. J. Birgeneau, G. S. Brown, P. M. Horn, D. E. Moncton, and P. W. Stephens, Phys. Rev. Lett. 48, 104 (1982); P.W. Stephens, R. J. Birgeneau, P. A. Heiney, P. M. Hom, and D. E. Moncton, Phys. Rev. B (in press).

${ }^{15}$ R. J. Birgeneau, G. S. Brown, P. M. Horn, D. E. Moncton, and P. W. Stephens, J. Phys. C 14, L49 (1981). 